\title{
Classic Spotlight, 1967 and 1968: Articles of Significant Interest Selected from the Journal of Virology Archives by the Editors
}

ournal of Virology (JVI) will mark its 50th year of publishing in 2017. JVI has published more than 40,000 articles since the first issue in February 1967. To mark particularly noteworthy JVI articles over the years, 2017 issues will feature articles of significant interest selected from the archives by the editors. These Classic Spotlights will be chronological, and in this issue, we have selected articles from 1967 and 1968.

Isolation of Epstein-Barr Virus P3HR-1 from a Burkitt Lymphoma Cell Line

A subline of the P3 (Jijoye) Burkitt lymphoma (BL) cell line, designated P3HR-1, was isolated and propagated by Hinuma et al. (J Virol 1:1045-1051, 1967, http://jvi.asm.org/ content/1/5/1045.abstract). Up to $40 \%$ of the cells showed immunofluorescence with selected human sera and the presence of herpes-type virus particles. Subsequent studies over the past 4 decades by several groups showed that P3HR-1 was an Epstein-Barr virus (EBV)-positive BL cell line and that P3HR-1 was nontransforming, unlike the parent, Jijoye, because it contains a deletion in the EBNA-2-encoding region required for transformation. P3HR-1 also was found to generate subgenomic infectious particles that reactivate the standard viral genome from a state of latency. Its components have been termed heterogeneous (het) DNA and are composed of four noncontiguous regions of EBV DNA joined by intramolecular recombination events. Although P3HR-1 was considered to be a consequence of propagation in cell culture, deletions similar to P3HR-1 were reported in viruses found in other BL cell lines, clinical throat washings, and oral hairy leukoplakia lesions. While these EBV variants are nontransforming in vitro, their potential to alter the course of natural infection by reactivating latent virus should now be considered.

Induction of Specific Chromosome Breaks by Adenovirus Type 12 in Human Embryonic Kidney Cells

Nonrandom chromosomal breaks in chromosomes 1 and 17 were discovered in human embryonic kidney cells after infection with adenovirus type 12 (Ad12) by zur Hausen, as reported in Journal of Virology in 1967 (J Virol 1:1174-1185, 1967, http:// jvi.asm.org/content/1/6/1174.abstract). Further studies by Alan Weiner's group found that the four sites of Ad12-induced metaphase chromosome fragility coincided with the human U1 genes (RNU1), U2 genes (RNU2), U1 pseudogenes (PSU1), and 5 S genes (RN5S) (Z. Li, A. D. Bailey, J. Buchowski, and A. M. Weiner, J Virol 72:4205-4211, 1998, http://jvi.asm.org/content/72/5/4205.abstract) and that Ad12-induced fragility of the RNU2 locus requires U2 small nuclear RNA transcriptional regulatory elements, viral early functions, and activation of p53 (Z. Li, A. Yu, and A. M. Weiner, J Virol 72:41834191, 1998, http://jvi.asm.org/content/72/5/4183.abstract). The discovery of the chromosome breaks by zur Hausen laid the groundwork for uncovering the mechanism by which Ad12 induces locus-specific fragility of the RNU1, RNU2, and RN5S loci.

\section{Protective Effects of Immunity to Influenza Virus Neuraminidase}

Antibodies specific for influenza virus neuraminidase (NA) were discovered in mice following pulmonary influenza virus infection in a study by Schulman et al. (J. L. Schulman, M. Khakpour, and E. D. Kilbourne, J Virol 2:778-786, 1968, http:// jvi.asm.org/content/2/8/778.abstract). Neuraminidase antibody had a profound inhibi-
Citation American Society for Microbiology. 2017. Classic Spotlight, 1967 and 1968: Articles of significant interest selected from the Journal of Virology archives by the editors. J Virol 91:e0197216. https://doi.org/10.1128/JVI.01972-16. Copyright $\odot 2016$ American Society for Microbiology. All Rights Reserved. 
tory effect on virus replication in the lungs of mice challenged with strains of virus having homologous NA protein, even in the absence of hemagglutinating inhibiting antibody, and resulted in decreased pulmonary virus titers and lung lesions. These findings suggested that anti-NA immunity plays a significant role in protection against influenza virus. Nearly 50 years after this report, the majority of influenza vaccine strategies target the viral hemagglutinin, the immunodominant antigen on the surface of the influenza virion. In 2015, it was reported that monospecific anti-NA immune sera inhibited influenza virus hemagglutination and infection of MDCK cells, suggesting that NA antibodies can interfere with virus attachment (S. J. Halbherr et al., J Virol 89:1550 1563, 2015, https://doi.org/10.1128/JVI.02949-14), and vaccination with recombinant NA induced broad heterologous cross-protection against influenza virus infection in mice [T. J. Wohlbold et al., mBio 6(2):e02556-14, 2015, https://doi.org/10.1128/ mBio.02556-14]. These studies highlight the importance of the NA antigen for the development of next-generation influenza vaccines.

\section{Defectiveness of Interferon Production and of Rubella Virus Interference in Vero Cells}

In a 1968 report, Desmyter et al. (J. Desmyter, J. L. Melnick, and W. E. Rawls, J Virol 2:955-961, 1968, http://jvi.asm.org/content/2/10/955.abstract) found that rubella virus, which conveys resistance to superinfection by a large number of unrelated viruses, including Newcastle disease virus, Sendai virus, Sindbis virus, and echovirus 11, did not interfere with infection by these viruses in Vero cells, a line of African green monkey kidney cells, even in cultures where nearly every cell was infected with rubella virus. The interference did not affect the uptake and eclipse of the challenge viruses and thus was likely at an intracellular level. The interferon system was suspected, and interferon was previously found in tissue culture cells infected with rubella virus. Other cells of primate origin produced interferon and showed rubella virus interference. This study reported the finding that Vero cells are sensitive to the action of exogenous interferon but are defective in interferon production. The results indicate that rubella virus interference is linked to the capacity of the cell to produce interferon and that Vero cells do not produce this antiviral effector. 